\title{
Generation of nonlinear internal waves by flow over topography: Rotational effects
}

\author{
C. Yuan \\ School of Mathematical Sciences, Ocean University of China, Qingdao, 266100, China \\ R. Grimshaw (1) \\ Department of Mathematics, University College London, London WC1E 6BT, United Kingdom \\ E. Johnson (1) \\ Department of Mathematics, University College London, London WC1E 6BT, United Kindom
}

A. Whitfield

Capitalab, London E14 5HP, United Kingdom

(Received 15 November 2019; accepted 13 February 2020; published 3 March 2020)

\begin{abstract}
We use the forced Ostrovsky equation to investigate the generation of internal waves excited by a constant background current flowing over localized topography in the presence of background rotation. As is now well known in the absence of background rotation, the evolution scenarios fall into three cases, namely subcritical, transcritical, and supercritical. Here an analysis of the linearized response divides the waves into steady and unsteady waves. In all three cases, steady waves occur downstream but no steady waves can occur upstream, while unsteady waves can arise upstream only when there is a negative minimum of the group velocity. The regions occupied by the steady and unsteady waves are determined by their respective group velocities. When the background current is increased, the wave number of the steady waves decreases. In addition, the concavity (canyon or sill), the topographic width, and the relative strength of the rotation play an important role in the generation mechanism. Nonlinear effects modulate the wave amplitude and lead to the emergence of coherent wave packets. All these findings are confirmed by numerical simulations.
\end{abstract}

DOI: 10.1103/PhysRevE.101.033104

\section{INTRODUCTION}

The generation of nonlinear internal waves in a density stratified fluid has been intensively studied since the lee wave generation mechanism proposed by Maxworthy [1] (see the review articles Cai et al. [2], Jackson et al. [3], and Guo and Chen [4]). In the decades since then, there has been an accumulation of in situ data, radar images, and high-resolution numerical simulations. Our understanding has consequently expanded and several mechanisms have been identified. These are internal tidal beams impinging on the pycnocline (New and Pingree [5], Gerkema [6], and Akylas et al. [7]), resonant generation due to lateral small-scale bottom variation and topographic contractions (da Silva and Helfrich [8] and Grimshaw and Helfrich [9]), linear sinusoidal internal tide steepening (Smyth and Holloway [10] and Farmer et al. [11]), and generation excited by river plumes (Nash and Moum [12] and Matthews et al. [13]). For a comprehensive review, see Jackson et al. [3]. It is noteworthy that most generation mechanisms are specific manifestation of interactions between flow, especially the barotropic tide, and topography.

The effect of the Earth's rotation on the dynamics of these nonlinear internal waves has been developed since the late

\footnotetext{
*r.grimshaw@ucl.ac.uk
}

1990s. For instance, Gerkema [14] found that the rotation suppresses the disintegration of the internal tide which otherwise evolves into nonlinear internal waves. Helfrich [15] and Grimshaw and Helfrich [16] showed that in the presence of rotation, an initial large-amplitude solitary wave rapidly decays into inertia-gravity waves, and eventually the leading disturbance forms into a coherent wave packet. The emergence of this kind of nonlinear wave packet was also confirmed by the laboratory experiments conducted by Grimshaw et al. [17]. Farmer et al. [18] examined the competition between rotational effects and nonlinearity, and Yuan et al. [19] demonstrated the rotational effects impacting on internal undular bores. While most attention so far has been concentrated on the effects of rotation on propagation, here we examine the role that rotation could also play on the generation of nonlinear internal waves.

The prominent generation mechanism is tide-topography interaction, and a fundamental assumption is a steady flow $U$ far upstream passing over a localized topography. The flow regime is then subcritical $\left(U<c_{0}, c_{0}\right.$ is the relevant linear long wave speed), supercritical $\left(U>c_{0}\right)$, or transcritical ( $U \approx c_{0}$, resonant generation). In the transcritical case nonlinearity has to be considered, and a useful model is the forced Korteweg-de Vries (fKdV) equation; see, for instance, Grimshaw and Helfrich [16], Grimshaw and Smyth [20], and Yuan et al. [21], among many others. In the absence of 


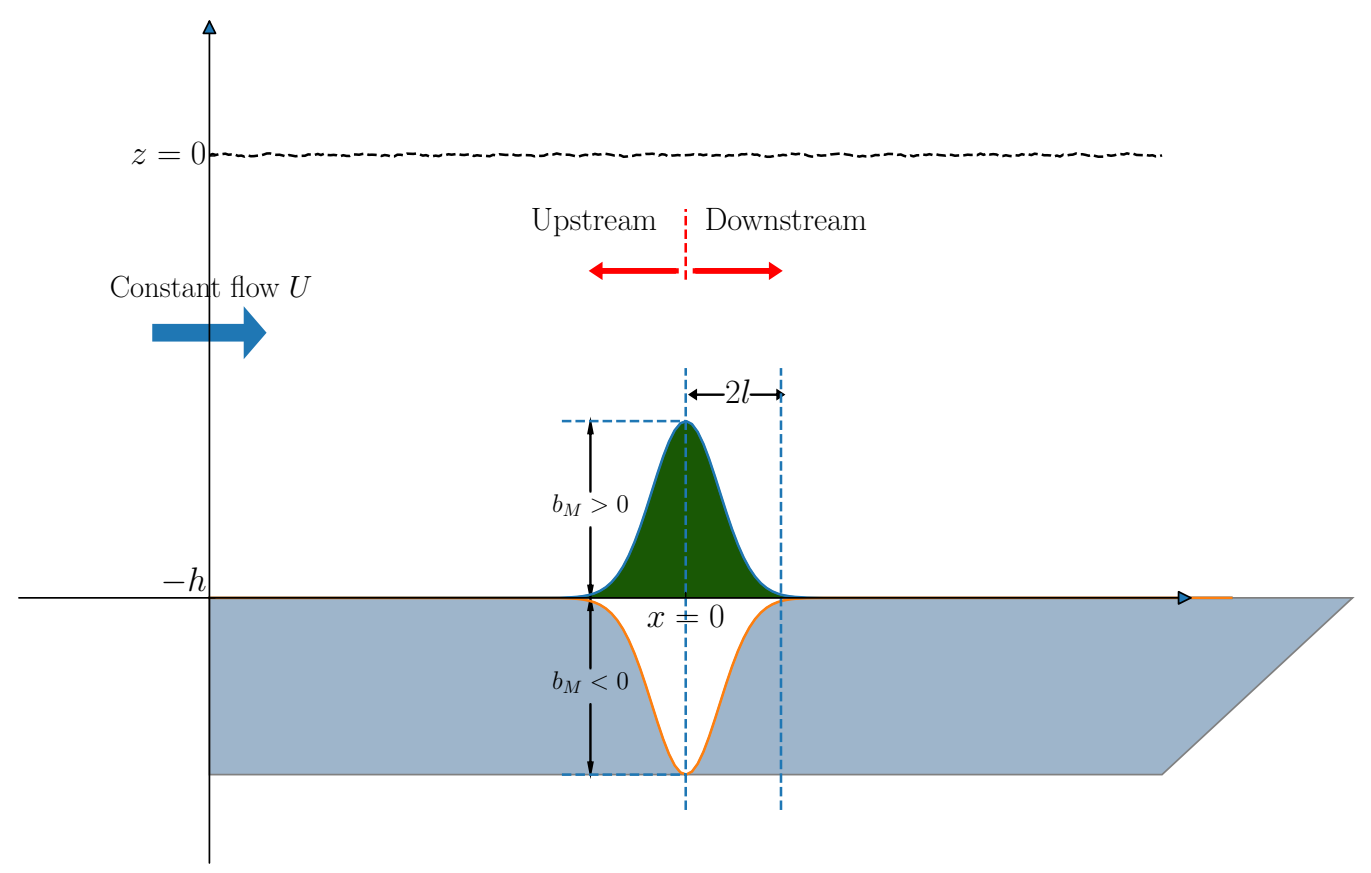

FIG. 1. The schematic diagram of the coordinate system. The Gaussian topography expressed as $b_{M} \exp \left[-(x / l)^{2}\right]$ where, given the pycnocline locates near the surface when the nonlinear coefficient $\mu_{0}<0$ [see Eq. (A13)], in reality, $b_{M}<0$ indicates undersea bump, for example, a sill or ridge, while $b_{M}>0$ implies undersea dent, for instance, a canyon or basin; refer to scale (A26).

rotation, the flow regime is characterized by a localized flow over topography and the generation of undular bores propagating both upstream and downstream. We note that comparison of models of KdV type with in situ data demonstrate satisfactory consistency for waves of small amplitude (Klymak et al. [22] and Yang et al. [23]) and still has value even for large-amplitude waves which are formally beyond the scope of the weakly nonlinear assumption of KdV theory; see Small et al. [24] and Ostrovsky and Stepanyants [25].

The purpose of this study is to investigate the generation of nonlinear internal waves by steady flow over localized topography in the presence of rotation. For this purpose we use the forced Ostrovsky equation, which is a combination of the fKdV equation (Grimshaw and Smyth [20] and Melville and Helfrich [26]) but with the rotational effect also considered, as in the Ostrovsky equation, which is an extension of the $\mathrm{KdV}$ equation, to allow for rotational effects, see Ostrovsky [27] and Grimshaw [28]. An analysis is conducted based on the linearized forced Ostrovsky equation in Sec. II, whose results are confirmed in Sec. III, together with the simulations including nonlinearity. We conclude and discuss in Sec. IV.

\section{LINEAR RESPONSE}

$\mathrm{KdV}$-type models have continued to evolve since Korteweg and de Vries [29], and the derivation and implementation can be found in the literature; for instance, for internal waves see Gerkema [14], Melville and Helfrich [26], Ostrovsky [27], Grimshaw [28], Benney [30], Benjamin [31], Grimshaw et al. [32,33], and Grimshaw and Helfrich [9] and the references therein.

Here the forced Ostrovsky equation is used to model internal waves excited by a background flow passing over a localized topography in the presence of the Earth's rotation.
It is a development from the $\mathrm{KdV}$ equation by adding both a forcing term and a rotational term. Its canonical form in nondimensional variables can be written as

$$
\frac{\partial}{\partial x}\left(-\frac{\partial A}{\partial t}-\Delta \frac{\partial A}{\partial x}+6 A \frac{\partial A}{\partial x}+\frac{\partial^{3} A}{\partial x^{3}}+F \frac{\partial b}{\partial x}\right)=\alpha A,
$$

where $x$ and $t$ represent space and time domain, respectively, and $A(x, t)$ is the wave amplitude. The Froude number $F=$ $U / c_{0}$ and a detuning parameter $\Delta=U-c_{0}\left[\Delta=c_{0}(F-1)\right]$ measure the ratio and difference of the constant background current $U$ to the intrinsic linear long wave speed $c_{0}\left(c_{0}\right.$ is obtained in the absence of a background flow). The localized topography is given by $b(x)$ and the rotational parameter $\alpha$ characterizes the effects of the Earth's rotation. Here we consider only the normal case for internal waves when $\alpha>0$. The coordinate system is sketched in Fig. 1. More details on the derivation of the forced Ostrovsky equation for internal waves can be found in the Appendix.

In the absence of rotation, Grimshaw and Smyth [20] and Grimshaw and Helfrich [9], based on the forced $\mathrm{KdV}$ theory, found that the generation mechanism falls into three categories, subcritical $\Delta<\Delta_{m}<0$, transcritical $\Delta_{m}<\Delta<$ $\Delta_{M}$, and supercritical $\Delta>\Delta_{M}>0$, where

$$
\Delta_{M, m}=6\left|b_{M}\right| \pm\left\{12\left|b_{M}\right|+36 b_{M}^{2}\right\}^{1 / 2}
$$

where $b_{M}$ is the magnitude of the localized forcing, which could be either positive or negative, depending on the topography, see Fig. 1. For long-time simulations, a rescaling is useful

$$
A=\alpha^{1 / 2} \bar{A}, x=\alpha^{-1 / 4} \bar{x}, t=\alpha^{-3 / 4} \bar{t}, \Delta=\alpha^{1 / 2} \bar{\Delta}, b=\alpha \bar{b} .
$$


Then Eq. (1) becomes, after removing the overbars,

$$
\begin{aligned}
& \frac{\partial}{\partial x}\left(-\frac{\partial A}{\partial t}-\Delta \frac{\partial A}{\partial x}+6 A \frac{\partial A}{\partial x}+\frac{\partial^{3} A}{\partial x^{3}}+F \frac{\partial b}{\partial x}\right)=A, \\
& F=1+\alpha^{1 / 2} \Delta .
\end{aligned}
$$

Note that the rotational parameter $\alpha$ appears only in the rescaled $F$. Thus, small $\alpha$ corresponds to large $b$ in this rescaled equation, generating large $A$. Also small $\alpha$ corresponds to large $\Delta$, and the boundaries of the transcritical regime (2) become

$$
\Delta_{M, m}=6 \alpha^{1 / 2}\left|b_{M}\right| \pm\left[12\left|b_{M}\right|+36 \alpha b_{M}^{2}\right]^{1 / 2} .
$$

In the limit as $\alpha \rightarrow 0$ this is unchanged.

In the absence of the forcing term, the linear dispersion relation for (1) is

$$
\omega(k)=\Delta k+k^{3}-\frac{\alpha}{k},
$$

where $\omega$ is the wave frequency and $k$ is the wave number, so that the phase and group velocities are

$$
c(k)=\Delta+k^{2}-\frac{\alpha}{k^{2}}, \quad c_{g}(k)=\Delta+3 k^{2}+\frac{\alpha}{k^{2}} .
$$

This, when as normal $\alpha>0, c$ takes all values in the range $(-\infty, \infty)$ as $k$ varies from zero to infinity, but $c_{g}-\Delta$ can only take positive values and has a minimum when $k=k_{m}$, $3 k_{m}^{4}=\alpha$. Hence all small waves with large $k$ propagate in the positive $x$ direction. In contrast a free solitary wave solution when $\alpha=0$ is given by

$$
A=a \operatorname{sech}^{2}[K(x-V t)], \quad V-\Delta=-2 a=-4 K^{2},
$$

and propagates in the negative $x$ direction at criticality when $\Delta=0$.

The linearized forced equation [(1) with the nonlinear term $A A_{x}$ omitted] can be solved exactly, and the solution consists of steady lee waves and some transients. The steady waves are found by setting $c=0$ and then $k= \pm k_{s}$, where

$$
\begin{aligned}
& \Delta+k_{s}^{2}-\frac{\alpha}{k_{s}^{2}}=0, \quad 2 k_{s}^{2}=-\Delta+\sqrt{4 \alpha+\Delta^{2}}, \\
& c_{g}=2 \sqrt{4 \alpha+\Delta^{2}} .
\end{aligned}
$$

Unlike the nonrotating case in which steady lee waves only occur when the flow is subcritical, it is clear that here steady lee waves can be found for all $\Delta$, and in all scenarios the group speed $c_{g}>0$, so they are always found downstream, $x>0$. As $\Delta$ increases, the wave number $k_{s}$ decreases, and even at exact resonance $\Delta=0$, there is a steady solution with $k_{s}^{4}=\alpha$. For large positive $\Delta, k_{s}^{2} \sim \alpha / \Delta$ and thus very long waves emerge, and for large negative $\Delta, k_{s}^{2} \sim-\Delta$ independent of $\alpha$.

The initial value problem for the linearized forced Ostrovsky equation with a zero initial condition can be solved with a Fourier transform,

$$
\begin{aligned}
A_{\operatorname{lin}}(x, t) & =\frac{1}{2 \pi} \int_{-\infty}^{\infty} \hat{A}(k, t) \exp (i k x) d k, \\
\hat{A}(k, t) & =\frac{k F \hat{b}(k)}{\omega(k)}\{1-\exp [-i \omega(k) t]\}, \\
\hat{b}(k) & =\int_{-\infty}^{\infty} b(x) \exp (-i k x) d x .
\end{aligned}
$$

This is split into a steady part and a transient part,

$$
\hat{A}_{\text {steady }}=\frac{k F \hat{b}(k)}{\omega(k)}, \quad \hat{A}_{\text {unsteady }}=-\frac{k F \hat{b}(k)}{\omega(k)} \exp [-i \omega(k) t] .
$$

It is then necessary to examine how the poles at $\omega(k)=0$, $k= \pm k_{s}$ are resolved. This is achieved in the usual way by adding a small imaginary part $i \epsilon, \epsilon>0$, to $\omega$. This has the effect that the poles at $\omega=0$ are displaced to $\omega=i \epsilon$. Then each pole in the complex $k$ plane is displaced to $k_{s}+i \delta$, where $c_{g}\left(k_{s}\right) \delta=\epsilon$. Since $c_{g}\left(k_{s}\right)>0$, the poles lie above the real $k$ axis. When $x<0$, the contour is displaced into the lower half-plane, and so $A_{\text {steady }}=0$ for $x \rightarrow-\infty$. But for $x>0$, the contour is displaced into the upper half-plane, and there is a contribution from the poles at $k= \pm k_{s}$, yielding

$$
A_{\text {steady }} \sim \frac{i k_{s} F \hat{b}\left(k_{s}\right)}{c_{g}\left(k_{s}\right)} \exp \left(i k_{s} x\right)+\text { c.c., } \quad x>0,
$$

where c.c. denotes the complex conjugate.

These lee waves are joined to the solution near the forcing site where $x \sim 0$. The effect of the obstacle width can be examined by assuming $b=b(X), X=x / l$. Then $\hat{b}=$ $l \hat{b}(K), K=l k$, and

$$
A_{\text {steady }}(x)=\frac{1}{2 \pi} \int_{-\infty}^{\infty} \frac{K F \hat{b}(K)}{l \omega(K / l)} \exp (i K X) d K .
$$

For wide forcing, $l \rightarrow \infty, \omega \approx-l \alpha / K$ and rotational effects are dominant. Evaluation of (13) shows that then the steady solution at the forcing site is given by

$$
A_{\text {steady }}(x) \approx \frac{F}{\alpha} \frac{\partial^{2} b}{\partial x^{2}} .
$$

On the other hand, for narrow forcing, $l \rightarrow 0$, when rotational effects are initially minimal, and there is no steady solution at the forcing site when $\alpha=0$ in the transcritical regime (2). Now $\omega \approx k^{3}$ and the integrand in (13) is proportional to $l^{2} / K^{2}$. The integral in (13) now diverges, but instead we note that $\partial_{x}^{2} A_{\text {steady }} \approx-F b$ in this limit.

A useful measure of nonlinearity vis-à-vis rotational effects is the Ostrovsky number $O_{s}$, defined as the ratio of the nonlinear term to the rotational term; see Farmer et al. [18] and Grimshaw et al. [34]. In the linear regime, there is a balance either between the linear dispersion and forcing, when $O_{s}=36 b_{M}^{2} F^{2} l^{4} / \alpha$, or between the rotational term and forcing, when $O_{s}=36 b_{M}^{2} F^{2} /\left(\alpha^{3} l^{4}\right)$. If the forcing is narrow, $l \rightarrow 0$, then the former relation holds and the rotational effect is small. However, if the forcing is wide, $\alpha^{3} l^{4} \sim 1$, then the rotational effect is dominant.

The unsteady part in (11) can be evaluated at large time using the method of stationary phase. The dominant contribution comes from $x / t=c_{g}(k)$ and is always in $x>\Delta t$ as $c_{g}>\Delta$ for all $k$, with dispersive decay as $t^{-1 / 2}$. For this unsteady part, the poles at $k= \pm k_{s}$ can be avoided by evaluating $\partial_{t} A$ instead of $A$ as then $\partial_{t} \hat{A}$ does not have any poles. Thus

$$
\begin{aligned}
& \frac{\partial A_{\text {unsteady }}}{\partial t}(x, t)=B(x, t)=\frac{1}{2 \pi} \int_{-\infty}^{\infty} \hat{B}(k, t) \exp (i k x) d k \\
& \hat{B}=-i k F \hat{b}(k) \exp [-i \omega(k) t] .
\end{aligned}
$$


TABLE I. The behaviors of the steady waves and unsteady waves in the subcritical, transcritical, and supercritical cases.

\begin{tabular}{lcc}
\hline \hline & Steady waves & Unsteady waves \\
\hline Subcritical & Wave number $k_{s}, 2 k_{s}^{2}=-\Delta+\sqrt{4 \alpha+\Delta^{2}}$. & The minimum group velocity $c_{g}\left(k_{m}\right)=\Delta+2(3 \alpha)^{1 / 2}$, \\
Transcritical & Group velocity $c_{g}=2 \sqrt{4 \alpha+\Delta^{2}}>0$. & either occur both upstream and downstream when \\
& Only occur downstream. & $c_{g}\left(k_{m}\right)<0$, or only occur downstream when $c_{g}\left(k_{m}\right)>0$. \\
Supercritical & $c_{g}\left(k_{m}\right)>0$ : only occur downstream. \\
\hline \hline
\end{tabular}

The method of stationary phase now gives

$$
\begin{aligned}
B(x, t) \sim & \sum-i k F \hat{b}(k)\left(\frac{1}{2 \pi|\varphi| t}\right)^{1 / 2} \\
& \times \exp \left\{i k x-i \omega(k) t+\operatorname{sign}[\varphi] \frac{i \pi}{4}\right\}+\text { c.c. } \\
\frac{x}{t}= & c_{g}(k), \quad \varphi=-\frac{\partial c_{g}}{\partial k}
\end{aligned}
$$

Here the sum is over the two positive solutions for $k$ of $x / t=c_{g}(k)$. The expression, however, is only valid provided that $\varphi \neq 0$, while near $\varphi=0$, this asymptotic solution is replaced by an Airy function. This unsteady solution exists only for $x / t>c_{g}\left(k_{m}\right)$, where $c_{g}\left(k_{m}\right)$ is the minimum value of $c_{g}$, defined by

$$
c_{g}\left(k_{m}\right)=\Delta+3 k_{m}^{2}+\frac{\alpha}{k_{m}^{2}}=\Delta+2(3 \alpha)^{1 / 2}, \quad 3 k_{m}^{4}=\alpha .
$$

It follows that transient waves can occur upstream for $\Delta<$ $-2(3 \alpha)^{1 / 2}$. A summary of these results is listed in Table I.

\section{NUMERICAL SIMULATIONS}

In order to check the analytical linear results in Sec. II against numerical simulations of the forced Ostrovsky equation, the pseudospectral method is implemented spatially, with a fourth-order Runge-Kutta iteration temporally. As the simulations are in a periodic domain, artificial damping layers are imposed at the two boundaries to prevent waves at one side from re-entering at the other side. To improve efficiency and accuracy, the simulations are based on the rescaled Eq. (4), and given the fact that the analytical results are originated from the form of (1), a transformation with scales (3) is conducted. A typical domain of length 2000 is discretized with 16384 grid cells, while the time step is $3.2 \times 10^{-3}$. The nondimensional topography is given by

$$
b(x)=b_{M} \exp \left[-\left(\frac{x}{l}\right)^{2}\right]
$$

where $b_{M}$ and $l$ are the height and characteristic width of the obstacle, respectively, see Fig. 1 for the schematic diagram.
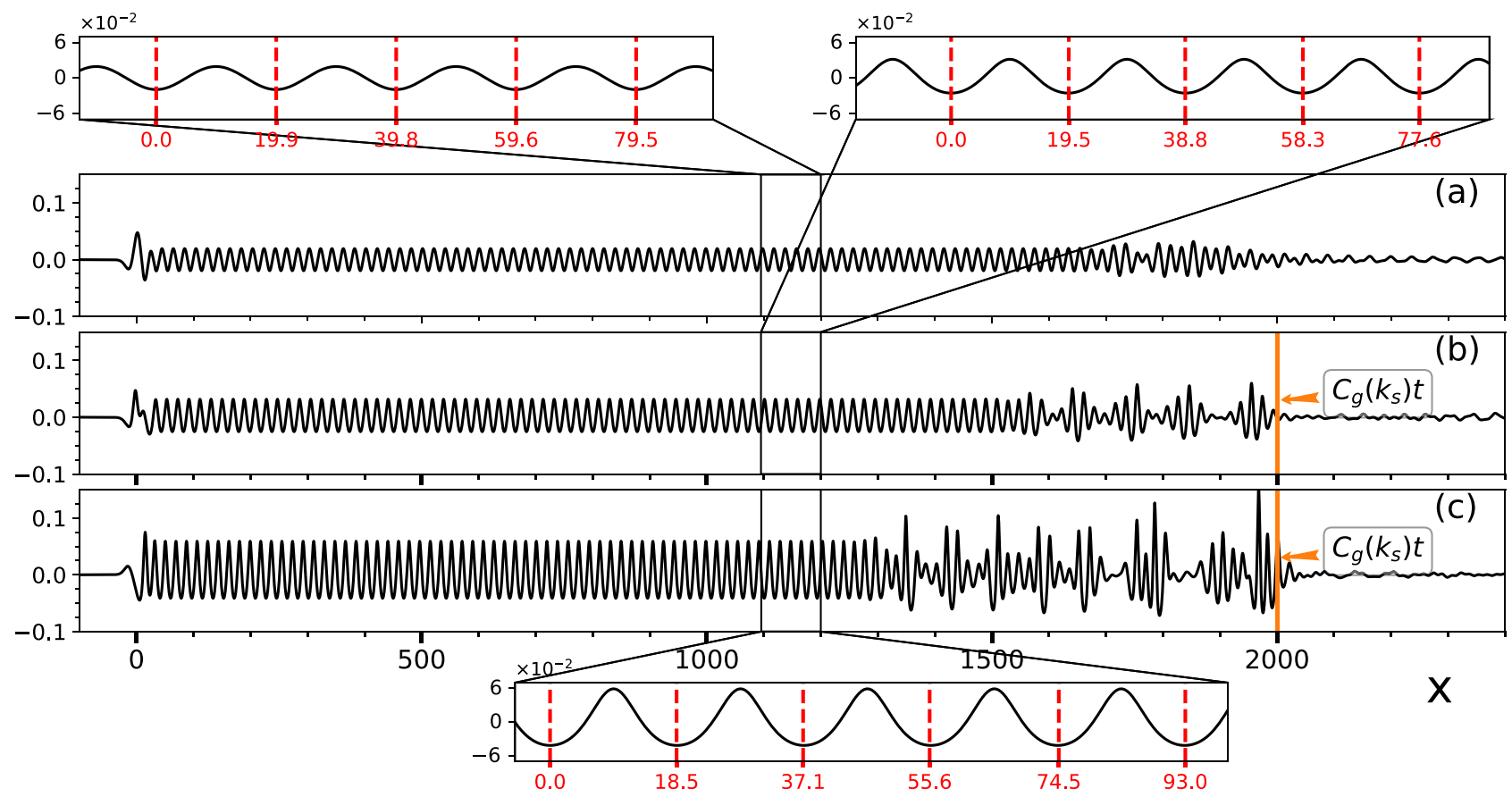

FIG. 2. Numerical simulations of the linearized (a) and full [(b) and (c)] Ostrovsky Eq. (4), while the results are shown in the scale of Eq. (1). These snapshots are captured at time $t=5000$. Here $b_{M}=-0.03$ for panels (a) and (b), $b_{M}=0.03$ for panel (c), and the other common parameters used are $(l, \Delta, \alpha)=(12.6,0,0.01)$, which indicates these scenarios locate on the transcritical case since the criteria $\Delta_{m}=-0.45$ and $\Delta_{M}=0.81$. 


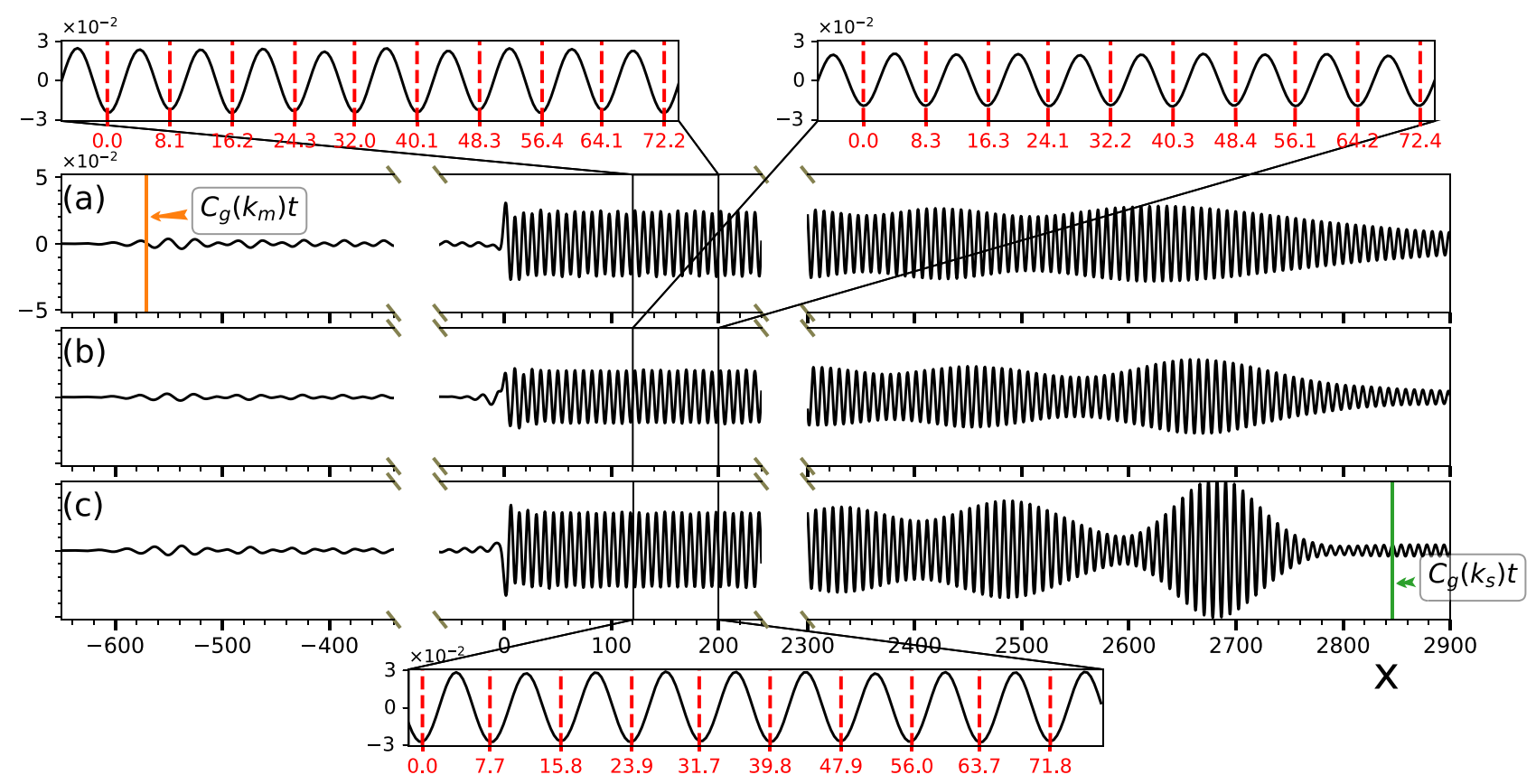

FIG. 3. The layout is the same as in Fig. 2. These snapshots are captured at time $t=2250$. Here $b_{M}=-0.04$ for panels (a) and (b), $b_{M}=0.04$ for panel (c), and the other common parameters used are $(l, \Delta, \alpha)=(3.2,-0.6,0.01)$, which indicates these scenarios locate on the subcritical case since the criteria $\Delta_{m}=-0.5$ and $\Delta_{M}=1.0$. To better illustrate the results, the axes are broken in the $x$ direction.

Figure 2 illustrates the scenarios of the wave generation when the background current is transcritical, where here $\Delta=0$ is chosen as an example. Figure 2(a) demonstrates the simulations of the linearized Ostrovsky equation, and at large times, it is clear that steady lee waves dominate the field near the topography, the linear wavelength is approximately 19.7, which matches with what the linear theory (9) predicts, $2 \pi / k_{s}=2 \pi / \alpha^{1 / 4}=19.9$. In the far field downstream, unsteady transient waves, asymptotically described by Eqs. (15) and (16) in the linear regime, are quite apparent and tend to evolve into wave packets but not very coherent. When the nonlinear effect is taken into account, see Fig. 2(b), the steady lee waves persist into the nonlinear regime as steady "cnoidal"-like waves with a slightly smaller wavelength and larger amplitude compared to their linear counterparts. Coherent nonlinear wave packets emerge in the far field, propagating with a speed approximately $c_{g}\left(k_{s}\right)(9)$, again, as noted in the linear theory. In this case, Eq. (16) indicates the transient unsteady waves only exist in the range of $x>c_{g}\left(k_{m}\right) t$, where $c_{g}\left(k_{m}\right)>0$ (17), thus no unsteady waves can occur upstream, and meanwhile, the steady lee waves cannot emerge upstream either [refer to Eq. (12)], so all waves propagate downstream.

Note that in the linear regime [(12) and (15), altering the sign of the forcing term $b$ can only reverse the polarity of the waves, but it gets more complicated when the nonlinear effect is considered. A comparison between Figs. 2(b) and Figs. 2(c) clearly illustrates that the nonlinear terms impose more effect on the case of positive forcing $b_{M}>0$, which appears to have more wave packets composed of waves with larger amplitude and smaller wavelength. In reality, for internal waves with a near-surface pycnocline, the nonlinear coefficient $\mu_{0}<0$ (A13), $b_{M}>0$ amounts to undersea canyon or basin. That is, the generation of large-amplitude internal waves is likely to be more efficient when the constant flow passes over holes rather than bumps.

When the background current is subcritical, $\Delta<0$, which allows unsteady transient waves upstream, as the group speed $c_{g}\left(k_{m}\right)=\Delta+2(3 \alpha)^{1 / 2}(17)$ has the possibility to be negative. In Fig. $3, \Delta=-0.6<\Delta_{m}=-0.5$, which falls into the subcritical case. Here the linear theory predicts that the unsteady waves propagate upstream with group speed $\left|c_{g}\left(k_{m}\right)\right|=0.25$ and the wavelength of the steady waves is 8.4 , which are both clearly confirmed by the simulations. Again, the case of positive forcing [Fig. 3(c)] generates larger nonlinear waves than that with negative forcing [Fig. 3(b)]. Distinguished from the transcritical cases, the nonlinear results with positive forcing forms coherent wave packets, whose group speed, however, is apparently overestimated in the linear theory. For the negative forcing, the linear results are larger than the nonlinear results, since here the nonlinear term takes on the effect of mitigation. Given a fixed background flow, there exists a criterion of the rotational parameter $\alpha$ determining the emergence of waves upstream. It is remarkable that when the group speed $c_{g}\left(k_{m}\right)=$ $\Delta+2(3 \alpha)^{1 / 2} \geqslant 0$, the waves will not propagate upstream, and this is examined in Fig. 4, where $c_{g}\left(k_{m}\right)=-0.25$ in Fig. 4(a) and $c_{g}\left(k_{m}\right)=0$ in Fig. 4(b). Clearly, the amplitudes in Fig. 4(b) are perceptively smaller than those in Fig. 4(a), which suggests that the rotational effect can suppress the generation and propagation of nonlinear internal waves, as pointed out by Farmer et al. [18], among many others. Note that in Fig. 4(a) with relatively weak rotation, the Ostrovsky number $O_{s}=1.3$ is larger than the critical value $O_{s c}=1$, and it indicates the formation of nonlinear internal waves; while $O_{s}=0.4<1$ in Fig. 4(b), and then the formation will be inhibited. 


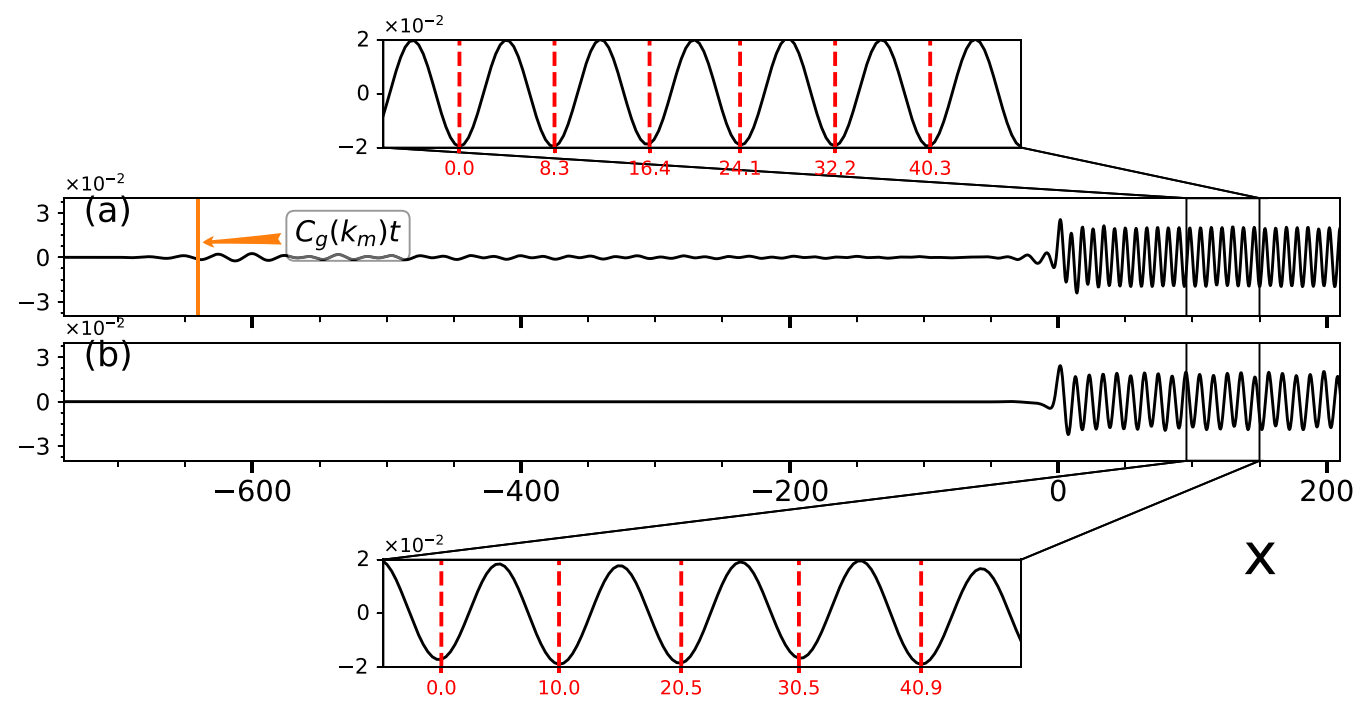

FIG. 4. The parameters used are $\left(b_{M}, l, \Delta\right)=(-0.04,3.2,-0.6)$, which indicates these scenarios locate on the subcritical case. Panel (a) indicates the rotation $\alpha=0.01$, while (b) for $\alpha=0.03$, and both are plotted at time $t=2500$.

For the supercritical case in Fig. 5, the basic dynamics is almost the same as in the aforementioned two cases: The simulated linear wavelength is the same as the prediction of Eq. (9); the results of positive forcing are larger than those of negative forcing. Nevertheless, no coherent wave packets occur in this case, which maybe needs a larger forcing term and long-time simulations. In Fig. 6, the influence of the width of the topography is examined. When the topography is wide, there are steady waves as described by Eq. (14), in contrast to the scenario when the topography is narrow.

\section{SUMMARY AND CONCLUSION}

Although there have been a large number of investigations into the generation mechanisms of oceanic internal solitary waves, the role of the Earth's background rotation has been less well examined, albeit we note some relevant studies: Farmer et al. [18], Gerkema and Zimmerman [35], and Ramirez and Renouard [36]. The barotropic tide interacting with localized topography is the most studied forcing term, especially in the coastal oceans, straits, and fjords. In this study we examine the effects of background rotation, using

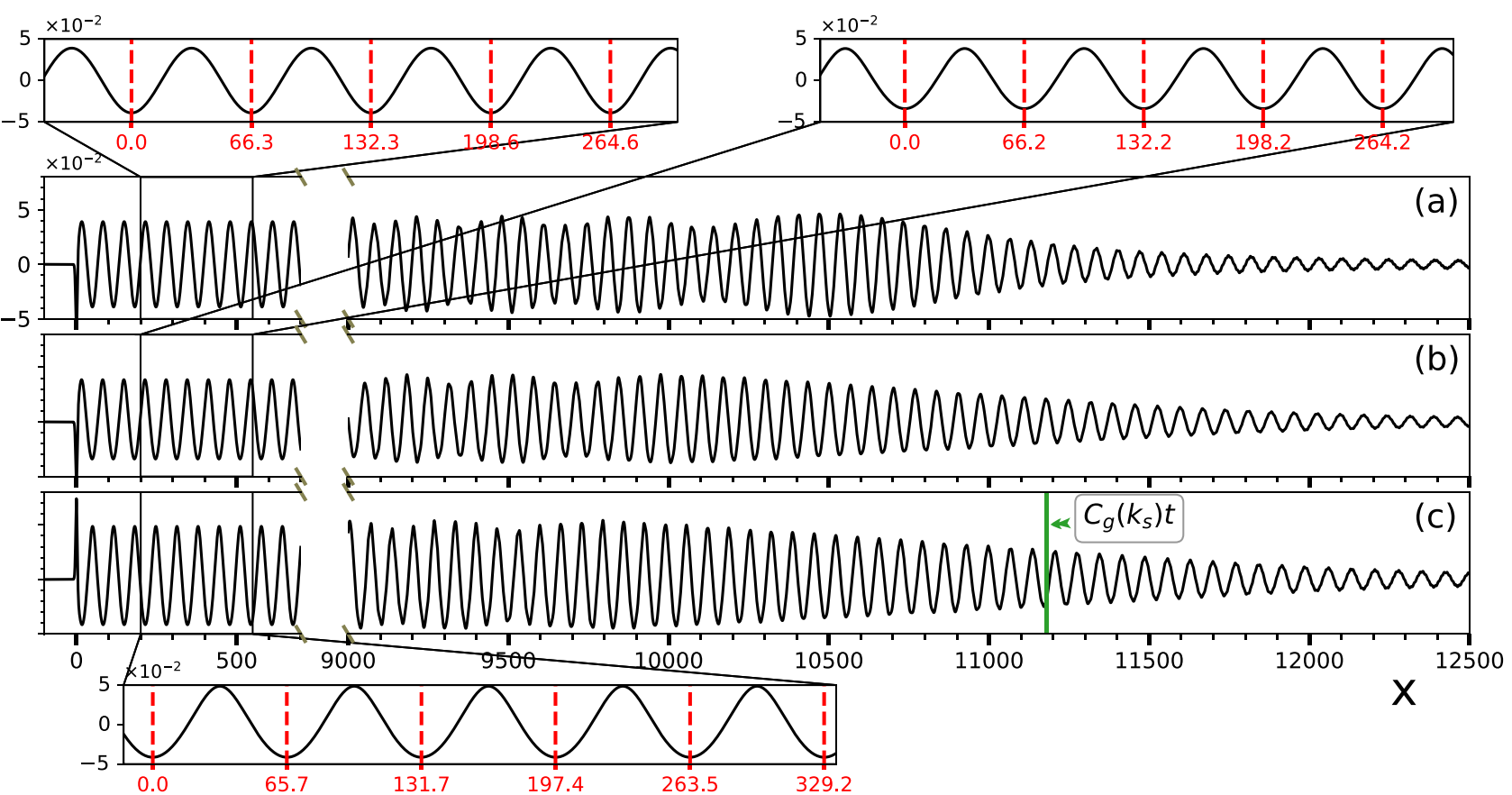

FIG. 5. The layout is the same as in Fig. 2. These snapshots are captured at time $t=5000$. Here $b_{M}=-0.04$ for panels (a) and (b), $b_{M}=0.04$ for panel (c), and the other common parameters used are $(l, \Delta, \alpha)=(3.2,1.1,0.01)$, which indicates these scenarios locate on the supercritical case since the criteria $\Delta_{m}=-0.5$ and $\Delta_{M}=1.0$. Note that the axes are broken in the $x$ direction. 

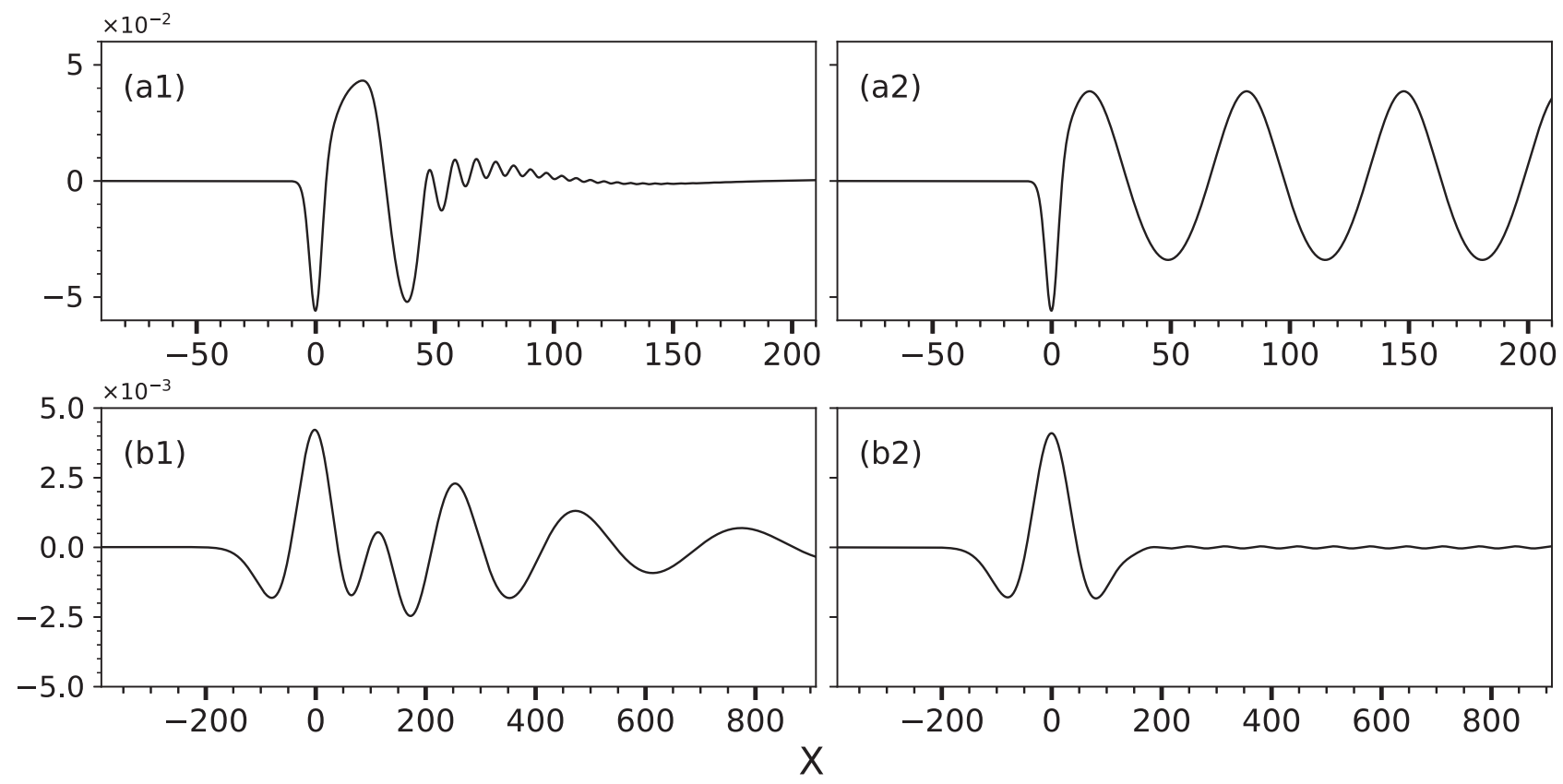

FIG. 6. The parameters used are $\left(b_{M}, \Delta, \alpha\right)=(-0.04,1.2,0.01)$, which indicates these scenarios locate on the supercritical case. Panels (a1) and (a2) indicate the width of topography $l=3.2$, while $l=70$ for (b1) and (b2), of which (a1) and (b1) are at time $t=25$ and (a2) and (b2) at time $t=5000$.

the forced Ostrovsky equation as the model, this being the rotational extension of the commonly used forced Kortweg-de Vries equation; see Grimshaw and Smyth [20]. As usual, we assume a constant background current as the forcing field.

Depending on the difference between background current $U$ and the intrinsic linear wave phase speed $c_{0}$, defining $\Delta=U-c_{0}$, the scenarios of evolution fall into three categories, namely subcritical when $\Delta<\Delta_{m}<0$, transcritical when $\Delta_{m}<\Delta<\Delta_{M}$, and supercritical when $\Delta>\Delta_{M}>0$; see Eq. (5). Linearized analysis demonstrates that steady lee waves can be generated for all values of $\Delta$, with larger $\Delta$ resulting in a larger wavelength, quite different from the nonrotating case when steady lee waves only arise in the subcritical regime. To facilitate the analysis, the waves are divided into steady and unsteady waves. For steady waves, the group velocity is always positive and so steady waves can only be found downstream. However, in the subcritical case unsteady waves can be found upstream when the minimum of group velocity $c_{g}\left(k_{m}\right)=\Delta+2(3 \alpha)^{1 / 2}<0$ where $3 k_{m}^{4}=\alpha$, defining the wave number where the minimum is reached. These analytical predictions are confirmed by the numerical simulations in Sec. III. In the absence of rotation, Grimshaw and Smyth [20] and Grimshaw and Helfrich [9] used the forced Korteweg-de Vries equation to show that in the transcritical regime, nonlinear waves occur both upstream and downstream, and relying on the strength of the forcing, their form can be a train of solitary waves of nearly uniform amplitude or undular bores modelled as a modulated cnoidal wave train. However, no such undular bores are found in the numerical results shown here. This is because the rotational effect acts to destroy the structure of undular bores and results instead in the emergence of nonlinear envelope wave packets; see Yuan et al. [19]. This demonstrates the potential crucial role of rotation on the generation mechanism.
When nonlinearity is taken into account, and the outcome compared with the linearized results, the most notable difference is the wave amplitude and the appearance of coherent nonlinear wave packets. In the transcritical cases, nonlinearity magnifies the wave amplitude, while in the other two cases, the nonlinear term mitigates the wave amplitude. This discrepancy in the amplitudes, conceivably, accounts for the slightly different wavelength and wave speed. In addition, it is found that the cases when $b_{M}>0$ (representing an undersea hole or canyon) are more efficient on the generation of internal waves than the case $b_{M}<0$ (undersea sill or ridge).

Finally, we examine the role of rotation on the generation mechanism for realistic ocean conditions. Luzon Strait in the South China Sea is a much-studied generation site, see Liu et al. [37], and much other literature for in situ observational data. At latitudes around $20^{\circ} \mathrm{N}$ the Coriolis parameter $f \approx$ $5 \times 10^{-5} \mathrm{~s}^{-1}$, and typical linear long wave speeds $c_{0}$ are approximately $2 \mathrm{~m} \mathrm{~s}^{-1}$. The coefficient $\delta_{0}=c_{0} h_{1} h_{2} / 6$ for a two-layer fluid model of upper and lower layer depths of $h_{1}, h_{2}$, from which we estimate that $\delta_{0} \approx 10^{3} \mathrm{~m}^{3} \mathrm{~s}^{-1}$ for shallow depths, $h_{1}=100 \mathrm{~m}, h_{2}=400 \mathrm{~m}$ (for the definitions of parameters refer to the Appendix). From Eq. (A27) this yields an estimate of the rotational coefficient $\alpha=\mathcal{O}\left(10^{-3}\right)$. This is smaller than the value used in our simulations, where we used a larger $\alpha$ to reduce computational time and to emphasize the role of rotation. But note that at higher latitudes and in deeper water $\alpha$ increases, and so rotational effects can be expected to increase; see Grimshaw et al. [38]. Transcritical flow requires that $\Delta=U-c_{0}$ is quite small, and although in the Luzon Strait the tidal forcing is usually subcritical on this criterion, see Grimshaw and Helfrich [9], we note that the transcritical regime increases with the amplitude of the topographic forcing. Further, in the present model we have assumed that the tidal current is a constant, whereas in reality 
it may vary on a timescale consistent with the rotational timescale. This aspect requires further study, as in Grimshaw and Helfrich [9], but with the rotational term included. In this present paper we have focussed on the simplest case of constant forcing to emphasize the rotational effects.

\section{ACKNOWLEDGMENTS}

C.Y. was supported by the Key Program of National Natural Science Foundation of China, Grant No. 91958206; China Postdoctoral Science Foundation, Grant No. 2019M660167; the Special Support for Post-doc Creative Funding in Shandong Province, Grant No. 201902021; and the Fundamental Research Funds for the Central Universities, Grant No. 201813024. R.G. was supported by a Leverhulme Emeritus Fellowship, EM-2019-030/9.

\section{APPENDIX: FORCED OSTROVSKY EQUATION}

In this Appendix we give a brief outline of the derivation of the forced Ostrovsky equation for internal waves. At the leading order we get from linear long wave theory,

$$
\zeta \sim A(x, t) \phi(z)+\cdots
$$

where $\zeta$ is the vertical particle displacement relative to the basic state defined by the density field $\rho_{0}(z)$ and background current $u_{0}(z)$, and $A(x, t)$ is the wave amplitude, see Fig. 1 for the coordinate system. The modal function $\phi(z)$ satisfies the system

$$
\begin{gathered}
\frac{\partial}{\partial z}\left[\rho_{0}\left(c-u_{0}\right)^{2} \frac{\partial \phi}{\partial z}\right]+\rho_{0} N^{2} \phi=0 \quad \text { for }-h<z<0, \\
\phi=0 \quad \text { at } z=-h, \quad\left(c-u_{0}\right)^{2} \frac{\partial \phi}{\partial z}=g \phi \quad \text { at } z=0
\end{gathered}
$$

where $h$ is the water depth, $g$ is the gravitational acceleration, and the buoyancy frequency $N(z)$ is defined by $N^{2}=-\frac{g}{\rho_{0}} \frac{d \rho_{0}}{d z}$. In general this has an infinite set of solution for $\phi(z)$ and the linear long wave speed $c$, but for oceanic internal waves it is customary to examine only mode one which has the fastest internal wave speed (formally the lowest mode zero is the surface wave mode with speed $c \approx \sqrt{g h}$ ). Then the forced Ostrovsky (fO) equation is written in the reference frame of the topography given by $z=-h+b(x)$,

$$
\begin{gathered}
\frac{\partial}{\partial x}\left[-\frac{\partial A}{\partial t}-c \frac{\partial A}{\partial x}+\mu A \frac{\partial A}{\partial x}+\delta \frac{\partial^{3} A}{\partial x^{3}}+\gamma \frac{\partial b}{\partial x}\right]=\Gamma A, \\
I \mu=3 \int_{-h}^{0} \rho_{0}\left(c-u_{0}\right)^{2}\left(\frac{\partial \phi}{\partial z}\right)^{3} d z \\
I \delta=\int_{-h}^{0} \rho_{0}\left(c-u_{0}\right)^{2} \phi^{2} d z \\
I \gamma=\rho_{0} u_{0}\left(u_{0}-c\right) \frac{\partial \phi}{\partial z}, \quad \text { at } z=-h, \\
I \Gamma=f^{2} \int_{-h}^{0} \rho_{0} \Phi \frac{\partial \phi}{\partial z} d z \\
\rho_{0}\left(c-u_{0}\right) \Phi=\rho_{0}\left(c-u_{0}\right) \frac{\partial \phi}{\partial z}-\frac{\partial\left(\rho_{0} u_{0}\right)}{\partial z} \phi \\
I=2 \int_{-h}^{0} \rho_{0}\left(c-u_{0}\right)\left(\frac{\partial \phi}{\partial z}\right)^{2} d z
\end{gathered}
$$

where $f=2 \Omega \sin \psi$ (where $\Omega$ is the rotation rate of the Earth and $\psi$ is the latitude) is the Coriolis parameter and $b(x)$ represents the localized obstacle, see Fig. 1. We consider a constant background current, $u_{0}=U=$ const, and then $c=$ $U-c_{0}$, where $c_{0}$ is the intrinsic linear long wave speed in the absence of a background flow and it can be obtained from Eqs. (A2) and (A3). At resonance (criticality) $c \approx 0$, and we define a detuning parameter $\Delta=c$. Equations (A4)-(A10) asymptotically reduce to

$$
\begin{gathered}
\frac{\partial}{\partial x}\left[-\frac{\partial A}{\partial t}-\Delta \frac{\partial A}{\partial x}+\mu_{0} A \frac{\partial A}{\partial x}+\delta_{0} \frac{\partial^{3} A}{\partial x^{3}}+\gamma_{0} \frac{\partial b}{\partial x}\right]=\Gamma_{0} A \\
I_{0} \mu_{0}=3 \int_{-h}^{0} \rho_{0} u_{0}^{2}\left(\frac{\partial \phi}{\partial z}\right)^{3} d z \\
I_{0} \delta_{0}=\int_{-h}^{0} \rho_{0} u_{0}^{2} \phi^{2} d z \\
I_{0} \gamma_{0}=\rho_{0} u_{0}\left(u_{0}-\Delta\right) \frac{\partial \phi}{\partial z}, \quad \text { at } z=-h \\
I_{0} \Gamma_{0}=f^{2} \int_{-h}^{0} \rho_{0} \Phi_{0} \frac{\partial \phi}{\partial z} d z \\
\rho_{0} u_{0} \Phi_{0}=\rho_{0} u_{0} \frac{\partial \phi}{\partial z}+\frac{\partial\left(\rho_{0} u_{0}\right)}{\partial z} \phi \\
I_{0}=2 \int_{-h}^{0} \rho_{0} u_{0}\left(\frac{\partial \phi}{\partial z}\right)^{2} d z
\end{gathered}
$$

Here the modal function $\phi$ is evaluated at $c=0$, but $c=\Delta$ is retained in the leading-order terms to ensure that all terms remain in balance.

Introducing the internal Froude number $F=U / c_{0}$, which is a dimensionless parameter in characteristics of the ratio of the background current to the linear phase speed, thus $\Delta=$ $c_{0}(F-1)$ and the modal equations (A2) and (A3) become

$$
\begin{gathered}
c_{0}^{2} \frac{\partial}{\partial z}\left(\rho_{0} \frac{\partial \phi}{\partial z}\right)+\rho_{0} N^{2} \phi=0, \quad \text { for } \quad-h<z<0, \\
\phi=0 \quad \text { at } \quad z=-h, \quad c_{0}^{2} \frac{\partial \phi}{\partial z}=g \phi \quad \text { at } \quad z=0 .
\end{gathered}
$$

The coefficients (A13)-(A17) become

$$
\begin{gathered}
I_{0} \mu_{0}=3 \int_{-h}^{0} \rho_{0} c_{0}^{2}\left(\frac{\partial \phi}{\partial z}\right)^{3} d z, \\
I_{0} \delta_{0}=\int_{-h}^{0} \rho_{0} c_{0}^{2} \phi^{2} d z, \\
I_{0} \gamma_{0}=\rho_{0} c_{0} U \frac{\partial \phi}{\partial z}, \quad \text { at } \quad z=-h, \\
I_{0} \Gamma_{0}=f^{2} \int_{-h}^{0} \frac{\partial\left(\rho_{0} \phi\right)}{\partial z} \frac{\partial \phi}{\partial z} d z, \\
I_{0}=2 \int_{-h}^{0} \rho_{0} c_{0}\left(\frac{\partial \phi}{\partial z}\right)^{2} d z .
\end{gathered}
$$

Note that in the Boussinesq approximation $\Gamma_{0} \approx f^{2} / 2 c_{0}$, which is always positive. In the fO Eq. (A11), the coefficients $\mu_{0}, \delta_{0}$ are independent of $F$, while the coefficients 
$\Delta=c_{0}(F-1)$ and $\gamma_{0}$ vary, where

$$
\gamma_{0}=F \sigma_{0}, \quad I_{0} \sigma_{0}=\rho_{0} c_{0}^{2} \frac{\partial \phi}{\partial z}, \quad \text { at } \quad z=-h
$$

To facilitate the following analyses and simulations, we rescale the variables as

$$
\begin{gathered}
\tilde{A}=\frac{\mu_{0} A}{6 c_{0}}, \quad \tilde{\Delta}=\frac{\Delta}{c_{0}}, \quad \tilde{t}=\left(\frac{c_{0}^{3}}{\delta_{0}}\right)^{1 / 2} t, \\
\tilde{x}=\left(\frac{c_{0}}{\delta_{0}}\right)^{1 / 2} x, \quad \tilde{b}=\frac{\sigma_{0} \mu_{0} b}{6 c_{0}^{2}},
\end{gathered}
$$

so that Eq. (A11) adopts the canonical form after removing the tilde superscript.

$$
\begin{aligned}
& \frac{\partial}{\partial x}\left(-\frac{\partial A}{\partial t}-\Delta \frac{\partial A}{\partial x}+6 A \frac{\partial A}{\partial x}+\frac{\partial^{3} A}{\partial x^{3}}+F \frac{\partial b}{\partial x}\right)=\alpha A, \\
& F=1+\Delta, \quad \alpha=\frac{\Gamma_{0} \delta_{0}}{c_{0}^{2}} \approx \frac{f^{2} \delta_{0}}{2 c_{0}^{3}} .
\end{aligned}
$$

Here we have used the Boussinesq approximation to get a convenient expression for $\alpha$. For mode-one internal waves, $\sigma_{0}>0$ and so the forcing $\tilde{b}(\tilde{x})$ is positive when $\mu_{0} b>0$ but negative when $\mu_{0} b<0$. In particular, flow of a two-layer fluid with a near-surface pycnocline over a sill is equivalent to negative forcing.
[1] T. Maxworthy, A note on the internal solitary waves produced by tidal flow over a three-dimensional ridge, J. Geophys. Res.: Oceans 84, 338 (1979).

[2] S. Cai, J. Xie, and J. He, An overview of internal solitary waves in the south china sea, Surv. Geophys. 33, 927 (2012).

[3] C. R. Jackson, J. C. B. Da Silva, and G. Jeans, The generation of nonlinear internal waves, Oceanography 25, 108 (2012).

[4] C. Guo and X. Chen, A review of internal solitary wave dynamics in the northern south china sea, Prog. Oceanogr. 121, 7 (2014).

[5] A. L. New and R. D. Pingree, Local generation of internal soliton packets in the central bay of biscay, Deep Sea Res. Part A 39, 1521 (1992).

[6] T. Gerkema, Internal and interfacial tides: Beam scattering and local generation of solitary waves, J. Mar. Res. 59, 227 (2001).

[7] T. R. Akylas, R. H. J. Grimshaw, S. R. Clarke, and A. Tabaei, Reflecting tidal wave beams and local generation of solitary waves in the ocean thermocline, J. Fluid Mech. 593, 297 (2007).

[8] J. C. B. da Silva and K. R. Helfrich, Synthetic aperture radar observations of resonantly generated internal solitary waves at Race Point Channel (Cape Cod), J. Geophys. Res.: Oceans 113, C11016 (2008).

[9] R. Grimshaw and K. R. Helfrich, Internal solitary wave generation by tidal flow over topography, J. Fluid Mech. 839, 387 (2018).

[10] N. F. Smyth and P. E. Holloway, Hydraulic jump and undular bore formation on a shelf break, J. Phys. Oceanogr. 18, 947 (1988).

[11] D. M. Farmer, M. H. Alford, R.-C. Lien, Y. J. Yang, M.-H. Chang, and Q. Li, From luzon strait to dongsha plateau: Stages in the life of an internal wave, Oceanography 24, 64 (2011).

[12] J. D. Nash and J. N. Moum, River plumes as a source of largeamplitude internal waves in the coastal ocean, Nature 437, 400 (2005).

[13] J. P. Matthews, H. Aiki, S. Masuda, T. Awaji, and Y. Ishikawa, Monsoon regulation of lombok strait internal waves, J. Geophys. Res.: Oceans 116, C05007 (2011).

[14] T. Gerkema, A unified model for the generation and fission of internal tides in a rotating ocean, J. Mar. Res. 54, 421 (1996).

[15] K. R. Helfrich, Decay and return of internal solitary waves with rotation, Phys. Fluids 19, 026601 (2007).
[16] R. Grimshaw and K. Helfrich, Long-time solutions of the ostrovsky equation, Stud. Appl. Math. 121, 71 (2008).

[17] R. H. J. Grimshaw, K. R. Helfrich, and E. R. Johnson, Experimental study of the effect of rotation on nonlinear internal waves, Phys. Fluids 25, 056602 (2013).

[18] D. Farmer, Q. Li, and J.-H. Park, Internal wave observations in the south china sea: The role of rotation and non-linearity, Atmos. Ocean 47, 267 (2009).

[19] C. Yuan, R. Grimshaw, and E. Johnson, The evolution of internal undular bores over a slope in the presence of rotation, Stud. Appl. Math. 140, 465 (2018).

[20] R. H. J. Grimshaw and N. Smyth, Resonant flow of a stratified fluid over topography, J. Fluid Mech. 169, 429 (1986).

[21] C. Yuan, R. Grimshaw, E. Johnson, and X. Chen, The propagation of internal solitary waves over variable topography in a horizontally two-dimensional framework, J. Phys. Oceanogr. 48, 283 (2018).

[22] J. M. Klymak, R. Pinkel, C.-T. Liu, A. K. Liu, and L. David, Prototypical solitons in the south China sea, Geophys. Res. Lett. 33, L11607 (2006).

[23] Y. Yang, Y. Fang, T. Tang, and S. Ramp, Convex and concave types of second baroclinic mode internal solitary waves, Nonlin. Process. Geophys. 17, 605 (2010).

[24] J. Small, Z. Hallock, G. Pavey, and J. Scott, Observations of large amplitude internal waves at the malin shelf edge during SESAME 1995, Continent. Shelf Res. 19, 1389 (1999).

[25] L. A. Ostrovsky and Y. A. Stepanyants, Internal solitons in laboratory experiments: Comparison with theoretical models, Chaos 15, 037111 (2005).

[26] W. K. Melville and K. R. Helfrich, Transcritical two-layer flow over topography, J. Fluid Mech. 178, 31 (1987).

[27] L. A. Ostrovsky, Nonlinear internal waves in a rotating ocean, Oceanology 18, 181 (1978).

[28] R. Grimshaw, Evolution equations for weakly nonlinear, long internal waves in a rotating fluid, Stud. Appl. Math. 73, 1 (1985).

[29] D. J. Korteweg and G. de Vries, On the change of form of long waves advancing in a rectangular channel, and a new type of long stationary wave, Phil. Mag. 39, 422 (1895).

[30] D. J. Benney, Long non-linear waves in fluid flows, J. Math. Phys. 45, 52 (1966).

[31] T. B. Benjamin, Internal waves of finite amplitude and permanent form, J. Fluid Mech. 25, 241 (1966). 
[32] R. Grimshaw, E. Pelinovsky, T. Talipova, and A. Kurkin, Simulation of the transformation of internal solitary waves on oceanic shelves, J. Phys. Oceanogr. 34, 2774 (2004).

[33] R. Grimshaw, E. Pelinovsky, T. Talipova, and O. Kurkina, Internal solitary waves: Propagation, deformation and disintegration, Nonlin. Proc. Geophys. 17, 633 (2010).

[34] R. Grimshaw, C. Guo, K. Helfrich, and V. Vlasenko, Combined effect of rotation and topography on shoaling oceanic internal solitary waves, J. Phys. Oceanogr. 44, 1116 (2014).
[35] T. Gerkema and J. T. F. Zimmerman, Generation of nonlinear internal tides and solitary waves, J. Phys. Oceanogr. 25, 1081 (1995).

[36] C. Ramirez and D. Renouard, Generation of internal waves over a shelf, Dynam. Atmos. Oceans 28, 107 (1998).

[37] A. K. Liu, F.-C. Su, M.-K. Hsu, N.-J. Kuo, and C.-R. Ho, Generation and evolution of mode-two internal waves in the south china sea, Continent. Shelf Res. 59, 18 (2013).

[38] R. Grimshaw, J. da Silva, and J. Magalhaes, Modelling and observations of oceanic nonlinear internal wave packets affected by the earth's rotation, Ocean Model. 116, 146 (2017). 